\title{
Utrecht Interstitial Applicator Shifts and DVH Parameter Changes in 3D CT-based HDR Brachytherapy of Cervical Cancer
}

\author{
Dan Shi ${ }^{\circledR}$, Ming-Yuan He ${ }^{\star}$, Zhi-Peng Zhao, Ning Wu, Hong-Fu Zhao, Zhi-Jian \\ Xu, Guang-Hui Cheng*
}

\begin{abstract}
Background: For brachytherapy of cervical cancer, applicator shifts can not be avoided. The present investigation concerned Utrecht interstitial applicator shifts and their effects on organ movement and DVH parameters during 3D CT-based HDR brachytherapy of cervical cancer. Materials and Methods: After the applicator being implanted, CT imaging was achieved for oncologist contouring CTVhr, CTVir, and OAR, including bladder, rectum, sigmoid colon and small intestines. After the treatment, CT imaging was repeated to determine applicator shifts and OARs movements. Two CT images were matched by pelvic structures. In both imaging results, we defined the tandem by the tip and the base as the marker point, and evaluated applicator shift, including $\mathrm{X}, \mathrm{Y}$ and $\mathrm{Z}$. Based on the repeated $\mathrm{CT}$ imaging, oncologist contoured the target volume and OARs again. We combined the treatment plan with the repeated $\mathrm{CT}$ imaging and evaluated the change range for the doses of CTVhr D90, D2cc of OARs. Results: The average applicator shift was $\mathbf{- 0 . 1 6} \mathbf{~ m m}$ to $0.10 \mathrm{~mm}$ for $X, 1.49 \mathrm{~mm}$ to $2.14 \mathrm{~mm}$ for $Y$, and $1.9 \mathrm{~mm}$ to $2.3 \mathrm{~mm}$ for $\mathrm{Z}$. The change of average physical doses and EQD2 values in $\mathbf{G y \alpha} / \boldsymbol{\beta}$ range for CTVhr D90 decreased by $2.55 \%$ and $3.5 \%$, bladder D2ce decreased by $5.94 \%$ and $8.77 \%$, rectum D2ce decreased by $2.94 \%$ and $4 \%$, sigmoid colon D2ce decreased by $3.38 \%$ and $3.72 \%$, and small intestines D2cc increased by $3.72 \%$ and $10.94 \%$. Conclusions: Applicator shifts and DVH parameter changes induced the total dose inaccurately and could not be ignored. The doses of target volume and OARs varied inevitably.
\end{abstract}

Keywords: Brachytherapy - cervical cancer - applicator shifts - DVH Parameter

Asian Pac J Cancer Prev, 16 (9), 3945-3949

\section{Introduction}

Carcinoma of the uterine cervix is the most common malignancy to affect females in developing countries (Shen et al., 2014). In developing countries, it accounts for about 3.4 lakh new cases and 1.6 lakh deaths every year (Kvale et al., 1988). Either surgery or radiotherapy alone can be used to treat early stages of cervix cancer. And the combinations of external beam radiation and brachytherapy have become a standard treatment for locally advanced cervix cancer (Green et al., 2001; Patidar et al., 2012). The external beam portion of treatment encompasses treatment to the pelvic lymph nodes, parametria, and primary tumor, to a dose adequate to control microscopic disease. The addition of brachytherapy serves to boost the gross tumor, protected normal organs, and improves disease control and survival (Hanks et al., 1983; Coia et al., 1990; Logsdon and Eifel, 1999), which have made this technique popular during the last decade (Potter et al., 2011). The CT based 3D Brachytherapy treatment plan is widely used in China (Ren et al.,2010). For brachytherapy, applicator shifts and DVH parameter changes could not be avoided. Uncertainties in the applicator shifts will result in uncertainties in the DVH parameters (Lang et al., 2013). Applicator positions can vary during treatment or the peroid between imaging and treatment. It could result in the dose varieties of target volume and normal organs. Besides, normal organ movement has some effects on primary tumor and normal organ (Ahamad et al., 2005). During the whole process of treatment, normal organ movement could not be avoided and would lead to applicator shift. In this article we investigate Utrecht intertitial applicator shifts and its effects on DVH parameters of cervical cancer during 3D CT-based high dose rate (HDR) brachytherapy.

\section{Materials and Methods}

\section{Patients and prescribed dose}

13 patients were included from January, 2013 to December, 2013 in our institute. Their ages range from 40 to 68 years old. All of the patients are diagnosed as squamous cancer. According to the tumor stage classification of the 


\section{Dan Shi et al}

International Federation of Gynecology and Obstetrics (FIGO), 7 patients had stage IIB, 3 had IIIA and 3 had IIIB. 6 patients received only two fractions of brachytherapy for their personal reasons.

External beam radiotherapy was performed for total dose to $45 \mathrm{~Gy} / 25$ fractions, cisplatin in 5-6 weekly cycles ( $40 \mathrm{mg} / \mathrm{m} 2$ ). The prescribed dose of HDR brachytherapy after external beam radiotherapy was $7 \mathrm{~Gy} \times 4$ fractions for 4 weeks. After the patients finished external beam radiotherapy, they received MR scanning in department of radiology before brachytherapy.

\section{Applicators}

Utrecht intertitial applicator sets used in the research are from Nucletron (Veenendaal, The Netherlands) a company of Elekta, Sweden. The tandem lengths were adjustable by changing the position of the base according to the depth of uterus with the maximum length for 60 $\mathrm{mm}$. The bending range of the tandem tip was 15 degrees and 30 degrees. Ovoid diameters varied from 15 to 20 $\mathrm{mm}$, and the angle between tandem and ovoid axis is 90 degree. Ovoids and tandem was fixed via the position of the base, and the distance between ovoid and tandem was immobilized.

\section{Imaging}

After the applicator being implanted, the CT imaging was achieved. The slice sickness was $2 \mathrm{~mm}$. The operation was made at the radiology department within one session in a fixed origin. We adopted the Zephyr Patient Transport Sled including Lithotomy Stirrups to minimize patient movement. The imaging data were exported to treatment planning system (TPS) and oncologist contoured the high risk CTV (CTVhr), the intermediate CTV (CTVir) and normal organs, including bladder, rectum, sigmoid colon and small intestines combined with the MR scanning before brachytherapy, which were performed according to GYN GEC ESTRO recommendations.

After that, CT imaging was repeated by the same slice sickness for $2 \mathrm{~mm}$. Oncologist contoured CTVhr, CTVir, and normal organs again.

\section{Treatment planning and optimization}

The contours were imported into Oncentra V4.3, Nucletron (Veenendaal, The Netherlands) a company of Elekta, Sweden and dose-volume-histogram (DVH) parameters for the standard plan were calculated and transformed to EQD2 values in Gy $\alpha / \beta$, biologically weighted dose normalized to $2 \mathrm{~Gy}$ fractionation (Kirisits et al., 2005; Lang et al., 2007). The used $\alpha / \beta$ values for target volumes and organ at risk (OARs) were 10 and 3 Gy, respectively. The contribution of the external beam dose distribution was also converted to EQD2 and added to the EQD2 DVH values of the brachytherapy plan.

The treatment plan was optimized according to the CTVhr, and executed in the case of total dose of CTVhr D90 controlled by 80 to $85 \mathrm{~Gy} \alpha / \beta$, and OARs dose constrained by $90 \mathrm{~Gy} \alpha / \beta 3$ for bladder, 75 Gy $\alpha / \beta 3$ for other organs.

\section{Applicator shifts and DVH parameter changes}

To evaluate the applicator shifts, the results of two CT imagings were matched by pelvic bony structures. In both imaging, we defined the tandem by the tip and the base as the marker point (Figure 1), and evaluated applicator shift, including $X$ (left as negative and right as positive), $\mathrm{Y}$ (head as negative and foot as positive) and Z (anterior as negative and posterior as positive).

Moreover, to evaluate the normal organ movement, oncologist contoured the target volume and OARs again based on the repeated CT imaging. We combined the treatment plan with the repeated CT imaging and evaluated the total dose of the target and OARs. We evaluated the change range for the doses of CTVhr D90, D2cc of OARs.

\section{Results}

Applicator shifts

Table.1 showed the shifts of tip and base of the tandem, the average, the standard deviation and the range in the 40 fractions. We found that both tip and base of the tandem shifted towards head and anterior direction on average. The left-right direction shift is relatively small in comparison with others. The largest shift was $16.86 \mathrm{~mm}$ in posterior direction (Figure 2 and $3 \mathrm{~A}$ ).

\section{DVH Parameter hangs}

The changes of the DVH parameters for CTVhr D90, D2cc of bladder, rectum, sigmoid colon and small intestines (Figure 3B), and the average, the standard deviation and the range in the 40 fractions were given in Table 2 and Table 3 . The variation percentage for 40 fractions was expressed by Gy and $\mathrm{Gy} \alpha / \beta$. The changes in dose to the CTVhr D90 were decreased by $2.55 \%$ on average for per fraction and $3.5 \%$ for $G y \alpha / \beta$. The change
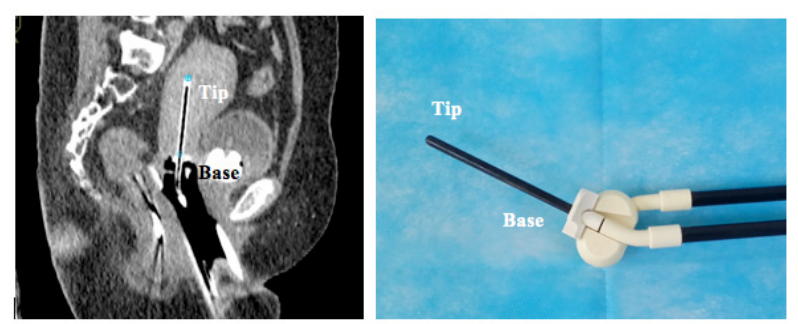

Figure 1. CT Images and Applicator set with Marker Position at Tip and Base of Tandem for Determining Applicator Shift

Table 1. Applicator Shifts Results for the Tip and Base of Tandem in mm Relation to Pelvic Structures. The Average, Standard Deviation (SD) and Range of 40 Fractions in 3 Directions are Shown in the Table

\begin{tabular}{lccccccccc}
\hline & \multicolumn{3}{c}{$\mathrm{X}(\mathrm{to}$ left)mm } & \multicolumn{3}{c}{$\mathrm{Y}$ (to head)mm } & \multicolumn{3}{c}{$\mathrm{Z}(\mathrm{to}$ anterior) mm } \\
& Average & SD & Range & Average & SD & Range & Average & SD & Range \\
\hline $\operatorname{Tip}(\mathrm{n}=40)$ & 0.10 & 1.90 & -4.51 to 4.31 & 1.49 & 3.98 & -8.67 to 9.51 & 2.30 & 4.80 & -6.75 to 16.86 \\
$\operatorname{Base}(\mathrm{n}=40)$ & -0.16 & 1.08 & -2.49 to 2.00 & 2.14 & 3.95 & -7.35 to 10.30 & 1.90 & 3.16 & -4.77 to 12.17 \\
\hline
\end{tabular}



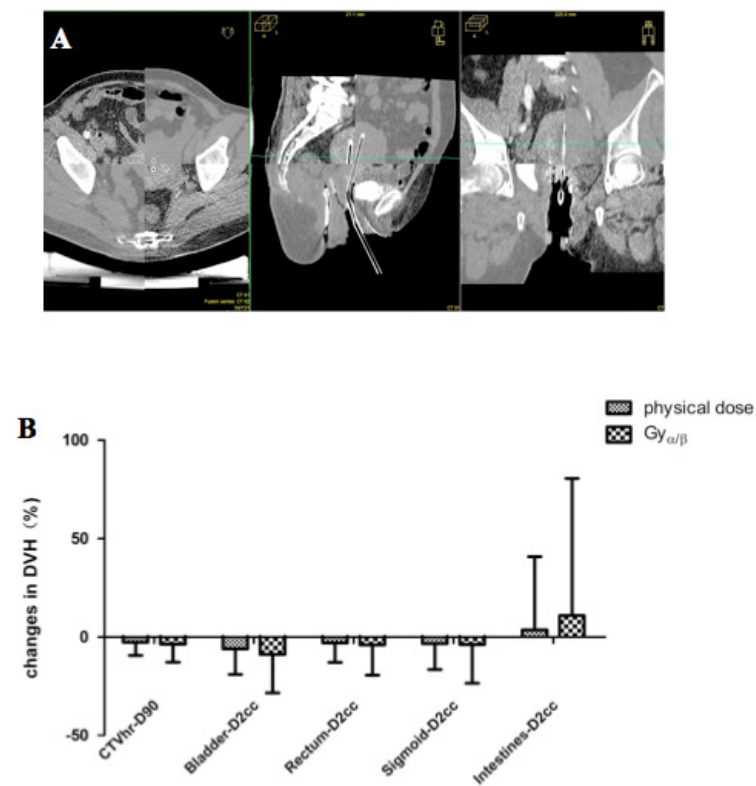

Figure 3. Applicator Shifts. (A) and DVH Parameter changes (B) after two matched CT images by pelvic bony structures
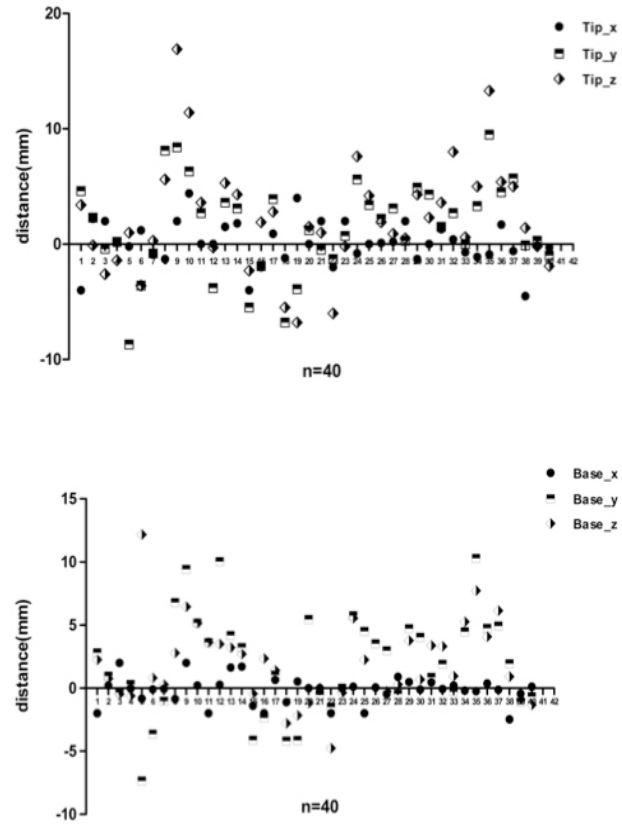

Figure 2. Applicator Shifts of the Tip and the Base of the Tandem

Table 2. CTV-hr D90 and Bladder D2cc Changes of two CT Images

\begin{tabular}{lcccccc}
\hline & & CTV-hr D90 $(\%)$ & \multicolumn{3}{c}{ Bladder D2cc(\%) } \\
& Average & SD & Range & Average & SD & Range \\
\hline Per fraction $(\mathrm{n}=40)$ & -2.55 & 6.73 & -11 to 16 & -5.94 & 12.95 & -40 to 11 \\
Per fraction in $\mathrm{Gy} / \boldsymbol{\beta}(\mathrm{n}=40)$ & -3.50 & 9.36 & -15 to 23 & -8.77 & 19.68 & -58 to 18 \\
\hline
\end{tabular}

Table 3. D2cc Changes of Rectum, Sigmoid Colon and Small Intestines After two CT Images. The average standard Deviation(SD) and the Range of 40 Fractions Expressed in \% of Physical dose, and in Gy $\alpha /$ /are Shown in the Table

\begin{tabular}{lccccccccc}
\hline & \multicolumn{3}{c}{ Rectum D2cc(\%) } & \multicolumn{3}{c}{ Sigmoid D2cc(\%) } & \multicolumn{3}{c}{ Small intestines D2cc(\%) } \\
& Average & SD & Range & Average & SD & Range & Average & SD & Range \\
\hline Per fraction $(\mathrm{n}=40)$ & -2.94 & 9.90 & -26 to 17 & -3.38 & 12.98 & -28 to 27 & 3.72 & 37.18 & -40 to 111 \\
Per fraction in $\mathrm{Gy} \alpha / \beta(\mathrm{n}=40)$ & -4 & 15.23 & -38 to 29 & -3.72 & 19.70 & -36 to 39 & 10.94 & 69.54 & -54 to 242 \\
\hline
\end{tabular}

for CTVhr D90 ranged between 11\% promotion and $16 \%$ decline for per fraction and between $15 \%$ decline and $23 \%$ rise for Gy $\alpha / \beta$. The change in dose to D2cc for bladder, rectum, sigmoid colon and small intestines were on average $5.94 \%$ decrease, $2.94 \%$ decrease, $3.38 \%$ decrease and $3.72 \%$ increase for per fraction and $8.77 \%$ decrease, $4 \%$ decrease, $3.72 \%$ decrease and $10.94 \%$ increase for Gy $\alpha / \beta$, respectively. The change for D2cc of bladder, rectum, sigmoid and small intestines ranged between $40 \%$ decrease and $11 \%$ increase, $26 \%$ decrease and $17 \%$ increase, $28 \%$ decrease and $27 \%$ increase, and $40 \%$ decrease and $111 \%$ increase for per fraction, and between $58 \%$ decrease and $18 \%$ increase, $38 \%$ decrease and $29 \%$ increase, $36 \%$ decrease and $39 \%$ increase, and $54 \%$ decrease and $242 \%$ increase for Gy $\alpha / \beta$.

\section{Discussion}

Brachytherapy forms an integral part of radiotherapy and cornerstone for both local control and toxicity in treating cervical cancer patients (Mahantshetty et al., 2012). With the development of technology, lots of radiation oncology center adopted MR scan during brachytherapy for cervix cancer, because it could provide precious boundaries of mucosa, especially for uterus, vagina, rectum, and bladder, and also thus is a good choice for assessment of local tumor extension, so MR is becoming increasingly popular in brachytherapy treatment planning in other countries (Morgia et al., 2013). Moreover CT scans are also widely used at some centers for image guidance during gynecological brachytherapy. CT images have some limitations when visualizing the tumor boundary from the cervix, uterus, vagina, rectum and bladder, the disadvantage of obscure boundary limits the use of CT images in brachytherapy (Viswanathan et al., 2007). However it was reported that the overall dose coverage of the CTVhr D90 and CTVir D90 was unevenful between MR and CT (Krishnatry et al., 2012), but the OARs could be delineated better on MR than CT (Viswanathan et al., 2007; Eskander et al., 2010). But cervical cancer is a frequently-occurring disease in developing countries and the use of MR in routine practice is unrealistic in these countries (Mahantshetty et al., 2012). The most cancer centers in developing countries including China lack availability of MR scanning because of the economic burden on patients, which makes MR 
imaging guided treatment in brachytherapy less feasible. So in our department, we make CT imaging for cervix cancer patients, and evaluate the applicator shift and DVH parameter changes in HDR brachytherapy.

In this article, we found that both applicator shifts and DVH parameter changes during the process of treatment, which could induce the doses of target volume and OARs inaccurately. Actually, the DVH parameters in brachytherapy for cervical cancer was uncertain, because of contouring, applicator shifts and normal organ movements (Potter et al., 2008). Applicator shifts could occur even during the $20 \mathrm{~min}$ of scanning time. The CTVhr and OAR's in brachytherapy with a single intracavitary application using an intrauterine line source without vaginal tube or a vaginal ring. They were re-contoured and the day 1 plan was applied to the new geometry to determine revised estimates of the CTVhr D90 and the bladder, rectal and sigmoid D2cc values. They found CTVhr D90 was decreased, which was consistent with our study and we also found that D2cc of bladder, rectum and sigmoid colon were all decreased. Only D2cc for small intestines increased.

With the development of brachytherapy, several kinds of applicators are applied in cervix cancer and uncertainties still exit in every step during brachytherapy. For the applicator shift, a flexible Ralstron or a rigid geometry Rotterdam applicator applicators, the average shift was similar, around $10 \mathrm{~mm}$ for each point during HDR brachytherapy procedures. The flexible uterine tube cannot fix together with ovoid (Datta et al., 2003). That makes the uterine tube move towards two ovoids. This would be a possible reason for the applicator shift to the pelvis, and resulted in significant differences in DVH parameters. In the case of Fletcher type applicators, its plastic tubes can decrease the dummy signals during MR imaging. However, Astrid et al (De Leeuw et al., 2009) inserted Fletcher CT/MR applicator set for patient, whose tandem and ovoids are clamped together with the fixation mechanism, and MR sans were involved to get imaging after each PDR fraction on the other day and they found that on average the shift of both tip and base of the tandem is in the ventral and cranial direction. Moreover, they found on average the left-right shift is relatively small, although shifts up to $10 \mathrm{~mm}$ occurred. The largest shift was $33 \mathrm{~mm}$ in the ventral direction. Besides, Jeno et al (Palvolgyi, 2010) also adopted the Fletcher-Suit (FS) applicator to investigate the influence of applicator geometries on sagittal dose distribution. The tandem length in uterus and the ovoid separation were determined by the patient anatomy. The slight sagittal ovoids shift was between 5 and $10 \mathrm{~mm}$. FS applicator geometries with correct ovoid position and large ovoid separation provide acceptable sparing of organs at risk, while FS insertions with small ovoid separation or insertions with ovoids is shifted towards the bladder or rectum and thus needs further measurement to decrease the dose of organs at risk. For Fletcher applicator, the tandem and ovoids are clamped together with the fixation, the relative position of tandem and ovoids are fixed, which means the relationship of tandem and ovoids is defined. However, the applicator shift exists during the brachytherapy treatment. There will always be a time lag between imaging and irradiation, so applicator shift and organ movements are inevitable, which results in DVH parameter changes. The dose inaccuracy for D90 CTVhr and normal organs, e.g. bladder, rectum, sigmoid colon and small intestines, induces the dose uncertainties for target, and normal organs, which could enhance the side effect to the normal organs.

An report from 6 gynecology cancer centers of different countries, showed that the derived random uncertainties were lowest for the bladder (20\%) and the rectum (22\%) and highest for sigmoid (27\%) (Nesvacil et al., 2013). In these 6 centers, two centers gave brachytherapy for PDR, others for HDR. They repeated MRI/CT scaning from 5 hours to 20 days, and a single treatment plan was applied to multi-fractionated brachytherapy treatment. Repeated imaging was only used to modify the doses of normal organs. The possibility of target and organ movement, e.g. changes in applicator's location related to the variations of shape and/or filling status of organs at risk, occurring between two individual brachytherapy fractions or within the time of delivery of one fraction, plays a crucial role in the assessment of total treatment doses for multi-fractional brachytherapy treatment (Nesvacil et al., 2013). After several fractions, the tumor shrinkage results in normal organs move close to the applicator, which increase the normal organ dose. Therefore the data do not allow us to draw any conclusions about the frequency of re-imaging. Consequently, plan adaptation is necessary in general.

The Utrecht intertitial applicator, which fixes the tandem and ovoids fixed together. The base and the tip position of the tandem are obvious. In order to control the movement of patients, we adopted the Zephyr Patient Transport Sled included in Lithotomy Stirrups, which could always keep the patients in the same position. To evaluate the applicator shifts, two 3D-based CT images were matched by pelvic structures within two hours. We defined the tandem by the tip and the base as the marker point, and evaluated applicator shift. We found that on average the shift of both tip and base of the tandem is to head and anterior direction. The left-right direction shift is relatively small in comparison with others. We hold that the applicator shift existed even though the Zephyr Patient Transport Sled included in Lithotomy Stirrups was used, HDR in our study reduced the time for treatment than PDR, and the time for whole process, including inserting, imaging, contouring, planning and treating, was controlled within 2 hours. Normal organ anatomic positions to cervix, e.g. bladder, rectum, sigmoid colon, and small intestines have little effect on applicator from left to right. When these normal organ movements occur unavoidably, the applicator shift is to head and anterior direction, but the left-right direction shift is relatively small. Applicator shift induced the changes of the DVH parameters for CTVhr D90, D2cc of bladder, rectum, sigmoid colon and small intestines. However, whether normal organ movements induce applicator shift, and then induce the changes of DVH parameters, still need further investigation.

It is the first time to report the Utrecht intertitial applicator shifts in the HDR brachytherapy of cervical cancer. During HDR fractions, applicator shifts and normal organ movement cannot be predicted and avoided, 
which lead to the inaccuracy of total dose. Although we adopted the Zephyr Patient Transport Sled included in Lithotomy Stirrups, which reduced the movement of patients, the doses of target volume and OARs varied inevitably. So applicator shifts and organ movements during the process of treatment could not be ignored. There is no solution available for this highly complex problem of CT based brachytherapy. The only way to deal with this problem is to repeat CT imaging immediately before the HDR treatment.

\section{Acknowledgements}

This work was supported by the Bethune Medical Scientific Research Support Plan of Jilin University frontier interdisciplinary innovation project (2013107024), the fund of the Department of Science and Technology of Jilin Province (20090458), the fund of the Health and Family Planning Commission Project of Jilin Province (2014ZC054).

\section{References}

Ahamad A, D'Souza W, Salehpour M, et al (2005). Intensitymodulated radiation therapy after hysterectomy: comparison with conventional treatment and sensitivity of the normaltissue-sparing effect to margin size. Int J Radiat Oncol Biol Phys, 62, 1117-24.

Coia L, Won M, Lanciano R, et al (1990). The Patterns of Care Outcome Study for cancer of the uterine cervix. Results of the Second National Practice Survey. Cancer, 66, 2451-6.

Datta NR, Basu R, Das KJM, et al (2003). Problems in reporting doses and volumes during multiple high-doserate intracavitary brachytherapy for carcinoma cervix as per ICRU Report 38: a comparative study using flexible and rigid applicators. Gynecologic Oncology, 91, 285-92.

De Leeuw AA, Moerland MA, Nomden C, et al (2009). Applicator reconstruction and applicator shifts in $3 \mathrm{D}$ MRbased PDR brachytherapy of cervical cancer. Radiother Oncol, 93, 341-6.

Eskander RN, Scanderbeg D, Saenz CC, et al (2010). Comparison of computed tomography and magnetic resonance imaging in cervical cancer brachytherapy target and normal tissue contouring, Int J Gynecol Cancer, 20, 47-53.

Green JA, Kirwan JM, Tierney JF, et al (2001). Survival and recurrence after concomitant chemotherapy and radiotherapy for cancer of the uterine cervix: a systematic review and meta-analysis. Lancet, 358, 781-6.

Hanks GE, Herring DF, Kramer S (1983). Patterns of care outcome studies. Results of the national practice in cancer of the cervix. Cancer, 51, 959-67.

Kirisits C, Potter R, Lang S, et al (2005). Dose and volume parameters for MRI-based treatment planning in intracavitary brachytherapy for cervical cancer. Int J Radiat Oncol Biol Phys, 62, 901-11.

Krishnatry R, Patel FD, Singh P, et al (2012). CT or MRI for image-based brachytherapy in cervical cancer. Jpn J Clin Oncol, 42, 309-13.

Kvale G, Heuch I, Ursin G (1988). Reproductive factors and risk of cancer of the uterine corpus: a prospective study. Cancer Res, 48, 6217-21.

Lang S, Kirisits C, Dimopoulos J, et al (2007). Treatment planning for MRI assisted brachytherapy of gynecologic malignancies based on total dose constraints. Int J Radiat Oncol Biol Phys, 69, 619-27.
Lang S, Nesvacil N, Kirisits C, et al (2013). Uncertainty analysis for 3D image-based cervix cancer brachytherapy by repetitive MR imaging: assessment of DVH-variations between two HDR fractions within one applicator insertion and their clinical relevance. Radiother Oncol, 107, 26-31.

Logsdon MD, Eifel PJ (1999). Figo IIIB squamous cell carcinoma of the cervix: an analysis of prognostic factors emphasizing the balance between external beam and intracavitary radiation therapy. Int $J$ Radiat Oncol Biol Phys, 43, 763-75.

Mahantshetty U, Khanna N, Swamidas J, et al (2012). Transabdominal ultrasound (US) and magnetic resonance imaging (MRI) correlation for conformal intracavitary brachytherapy in carcinoma of the uterine cervix. Radiother Oncol, 102, 130-4.

Morgia M, Cuartero J, Walsh L, et al (2013). Tumor and normal tissue dosimetry changes during MR-guided pulsed-doserate (PDR) brachytherapy for cervical cancer. Radiother Oncol, 107, 46-51.

Nesvacil N, Tanderup K, Hellebust TP, et al (2013). A multicentre comparison of the dosimetric impact of inter- and intrafractional anatomical variations in fractionated cervix cancer brachytherapy. Radiother Oncol, 107, 20-5.

Palvolgyi J (2010). Influence of different Fletcher-Suit applicator geometries on sagittal dose distribution. Phys Med, 26, 49-54.

Patidar AK, Kumar HS, Walke RV, et al (2012). Evaluation of the response of concurrent high dose rate intracavitary brachytherapy with external beam radiotherapy in management of early stage carcinoma cervix. J Obstet Gynaecol India, 62, 562-5.

Potter R, Georg P, Dimopoulos JC, et al (2011). Clinical outcome of protocol based image (MRI) guided adaptive brachytherapy combined with 3D conformal radiotherapy with or without chemotherapy in patients with locally advanced cervical cancer. Radiother Oncol, 100, 116-23.

Potter R, Kirisits C, Fidarova EF, et al (2008). Present status and future of high-precision image guided adaptive brachytherapy for cervix carcinoma. Acta Oncol, 47, 132536.

Ren YF, Gao YH, Cao XP, et al (2010). 3D-CT implanted interstitial brachytherapy for $\mathrm{T} 2 \mathrm{~b}$ nasopharyngeal carcinoma. Radiat Oncol, 5, 113.

Shen XR, Feng R, Chai J, et al (2014). Modeling age-specific cancer incidences using logistic growth equations: implications for data collection. Asian Pac J Cancer Prev, 15, 9731-7.

Viswanathan AN, Dimopoulos J, Kirisits C, et al (2007). Computed tomography versus magnetic resonance imagingbased contouring in cervical cancer brachytherapy: results of a prospective trial and preliminary guidelines for standardized contours. Int J Radiat Oncol Biol Phys, 68, 491-8. 